\title{
Editorial: Quality of life after anterior skull base surgery
}

\author{
Brett E. Youngerman ${ }^{1} \cdot$ Theodore H. Schwartz ${ }^{1}$
}

Received: 5 September 2019 / Accepted: 9 September 2019 /Published online: 3 October 2019

(C) Springer-Verlag GmbH Austria, part of Springer Nature 2019

The present study by Wagner et al. should be commended for its effort to rigorously measure patient-reported quality of life (QOL) and emotional burden after anterior skull base surgery. The study reports well-validated, cross-population QOL measures such as EuroQol and SF-36. In addition, the authors collected an extensive battery of questionnaires to measure depression, anxiety, and post-traumatic stress. Such thoughtful and time-consuming data collection is far from routine in the surgical literature. Importantly, the study demonstrates progressive improvement in anxiety and depression scores at 3 months and 1 year after surgery compared to preoperative testing.

QOL measures are increasingly recognized as a critical component of surgical outcomes and are the cornerstone of patient-centered decision-making, research, and innovation. Surgeons can and should track clinical outcomes, such as vision and hormone function, radiographic outcomes, such as tumor residual and recurrence, and adverse events, such as sinusitis and CSF leak. However, only the patient can weigh the tradeoffs between these many outcomes and report the balance in terms of quality of life.

While QOL is clearly an important factor to be considered in surgical outcome, it remains to be determined how to weigh patient-centered concerns against more traditional metrics such as extent of resection, complications, and length of stay. For example, if a patient is relieved of pre-operative anxiety based on the surgeon's determination that the outcome was perfect yet only half the tumor was removed by a timid surgeon, and the patient stayed in the hospital 7 days instead of 3 days because of a urinary tract infection (UTI), is this an

This article is part of the Topical Collection on Brain Tumors

Theodore H. Schwartz

schwarh@med.cornell.edu

1 Department of Neurosurgery, Weill Cornell Medicine, New York Presbyterian Hospital, 525 East 68th St., Box \#99, New York, NY 10065, USA optimal outcome? As long as the patient has not been informed that their tumor has grown back in the first 3 or 12 months, they may have excellent QOL scores but be heading for another surgery, radiation, accompanying morbidity, and perhaps a decrease in overall survival in the next few years. Thus, we cannot look at QOL in isolation without weighing more traditional measures of surgical success to find the right balance.

The study's additional findings are limited by several challenges common to single-center QOL studies. First, the comparison between transnasal and open approaches is confounded by the center's chosen approach to each pathology; all of the transnasal approaches were for adenomas while the craniotomies were for meningiomas. This ignores significant differences between adenomas and meningiomas in terms of surgical location, difficulty, and morbidity, as well as symptoms and disease course. For example, the adenoma group had much higher rates of hormonal deficiency, which likely has an impact on psychological and QOL outcomes. Different factors may influence QOL in these different patient populations. We have previously shown that subtotal resection correlated with worse QOL in pituitary adenomas [1] while advanced age predicted less favorable patient-reported outcomes in endoscopic meningioma surgery [2]. After endoscopic surgery for craniopharyngiomas, the picture is even more complex and multiple factors, including visual deficits, hypopituitarism, weight gain, and recurrence, affect QOL [3]. Meaningful comparisons between different techniques can only be made when they are used to treat similar pathologies.

Second, while widely validated QOL scales are valuable for population-level comparisons and cost-effectiveness analysis, disease-specific measures may provide more information about what symptoms are driving overall QOL. We have shown that endoscopic skull base surgery is associated with an improvement in postoperative site-specific QOL as compared with the preoperative QOL [4]. Patients with adenomas and other pathologies experience progressive improvement on the Anterior Skull Base Questionnaire (ASBQ) [1, 4], while sinonasal QOL, as measured by the Sino-Nasal Outcome Test 
(SNOT-22), transiently declines and then returns to preoperative baseline levels $[1,4,5]$.

Finally, the high dropout rate (over 25\%) creates significant potential for bias. Psychological and QOL outcomes may influence a patient's likelihood of returning for follow-up or filling out a questionnaire and therefore confound results.

The study by Wagner et al. exemplifies both the importance and the challenge of reporting QOL with different surgical techniques. Where randomized trials are not feasible, multi-center observational studies and registries are needed to compare various concurrently practiced techniques across patients with similar pathologies. This requires a consistent collection of standardized QOL measures at routine follow-up intervals with minimal dropout. Generic and disease-specific QOL instruments may capture different aspects of the patient experience. Rigorous collection of patient and clinical characteristics is needed for risk-adjusted comparisons. Moreover, QOL data must be viewed alongside more traditional measures of surgical success and not be evaluated in isolation or else we throw out the baby with the bathwater. Such data collection is resource intensive and requires coordination between multiple centers, across disciplines, and with patients and families. However, the information is critical to better understand the true impact of surgical interventions on our patients.

\section{Compliance with ethical standards}

Conflict of interest None

\section{References}

1. McCoul ED, Bedrosian JC, Akselrod O, Anand VK, Schwartz TH (2015) Preservation of multidimensional quality of life after endoscopic pituitary adenoma resection. J Neurosurg 123(3):813-820. https://doi.org/10.3171/2014.11.JNS14559

2. Jones SH, Iannone AF, Patel KS et al (2016) The impact of age on long-term quality of life after endonasal endoscopic resection of skull base meningiomas. Neurosurgery. 79(5):736-745. https://doi.org/10. 1227/NEU.0000000000001360

3. Patel KS, Raza SM, McCoul ED et al (2015) Long-term quality of life after endonasal endoscopic resection of adult craniopharyngiomas. J Neurosurg 123(3):571-580. https://doi.org/ 10.3171/2014.12.JNS141591

4. McCoul ED, Anand VK, Schwartz TH (2012) Improvements in sitespecific quality of life 6 months after endoscopic anterior skull base surgery: a prospective study. J Neurosurg 117(3):498-506. https:// doi.org/10.3171/2012.6.JNS111066

5. McCoul ED, Anand VK, Bedrosian JC, Schwartz TH (2012) Endoscopic skull base surgery and its impact on sinonasal-related quality of life. Int Forum Allergy Rhinol 2(2):174-181. https://doi. org/10.1002/alr.21008

Publisher's note Springer Nature remains neutral with regard to jurisdictional claims in published maps and institutional affiliations. 